Health \& Medicine | Toshimitsu Yamaoka

\title{
Understanding the EGFR mutation aids the fight against lung cancer
}

Genetic mutations are known
to be responsible for several
different types of cancer. One
type, known as non-small cell
lung cancer, is sometimes
caused by mutations in a
gene called EGFR. A group of
drugs called tyrosine kinase
inhibitors are often used to
treat patients with this type
of cancer. Unfortunately,
patients can develop genetic
alterations, namely mutation,
amplification (an increase in the
number of copies of a gene) or
methylation (methyl groups are
added to the DNA molecule),
that trigger resistance to
these drugs. In-depth research
by Dr Toshimitsu Yamaoka
and colleagues at Showa
University, Tokyo, Japan, aims
to uncover the mechanisms of
drug resistance in lung cancer
patients so that new treatment
options can be explored.

enetic alterations have been lifetime, rather than being inherited 7 associated with many different gene, several different mutations have EGFR MUTATIONS been found to be linked with lung transmemally, EGRR, which is a their signals from the environments, then carries the instructions a cell need in order to adapt to the environment to the nucleus. The rol of the receptor protein is to bind to other proteins outside the cell, known as ligands, and to help the cell communicate with its environment.

Occasionally, the EGFR gene develops mutations that disrupt the function of the receptor protein. When this occurs, the receptor protein is effectively always 'on'. This means that the cell continuously receives signals that tell it to survive and, crucially, grow Mutations in EGFR a so a tum is, they develop during a person's

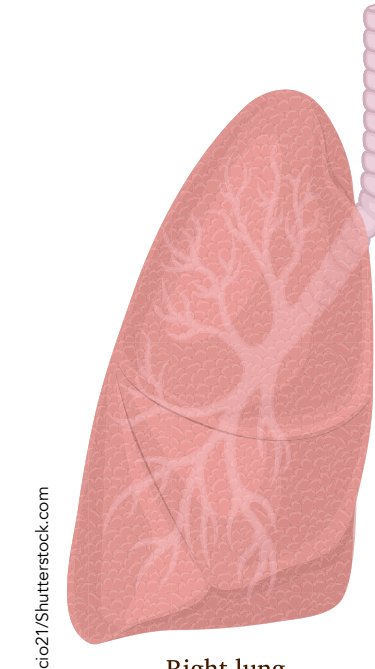

Right lung

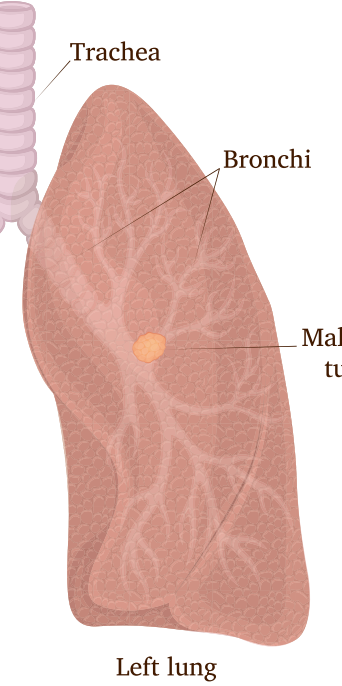

Non-small-cell
cancer cells

$$
\text { cancer cells }
$$
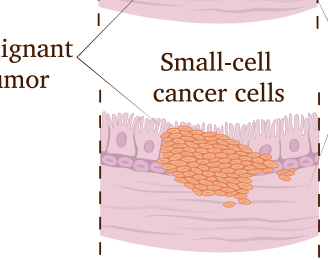
Unlike small cell lung cancer, non-small
cell lung cancer can sometimes occur
in non-smokers.

\section{LUNG CANCER}

Most mutations in EGFR trigger a

lung cancer. Non-small cell lung

cancer (which, unlike other types of

lung cancer, is weakly associated with

smoking) accounts for about $80-85 \%$ of

lung cancer cases in the UK. Mutations in EGFR are particularly common in

people of Asian ethnicity. Research has revealed that, among non-small cell

have mutations in the EGFR gene,

con patients of

Lung cancer caused by EGFR mutations is often treated with a EGFR tyrosine kinapy drugs called These drugs work by binding to the malfunctioning receptor proteins in the cell membrane, blocking their activity and therefore stopping the unchecked growth of the cell. This group of drug includes the medications gefitinib, erlotinib, afatinib, and osimertinib. When given to patients as a first-line treatment for non-small cell lung cancer, the response rate to these drugs is $70-80 \%$ (i.e. in $70-80 \%$ of patients, the tumour stops growing shrinks or disappears).

Unfortunately, most patients treated with EGFR TKIs develop resistance to the drugs after one to two years, to the appearas ls least partly due mutations in the EGFR gene. When this happens, the drugs are no longer secive and the palionts chances of patients often then face treatment with traditional, cytotoxic (i.e. celldestroying) chemotherapy, which is less effective and frequently causes severe side-effects.

UNDERSTANDING THE GENETICS OF DRUG RESISTANCE

Dr Toshimitsu Yamaoka of Showa

University, Japan, investigates the

complex relationships between

mutations in the EGFR gene, EGFR TKI drugs, and drug resistance in non-small cell lung cancer. The goal of Dr Yamaok and his colleagues is to overcome the problems generated by EGFR-mediated drug resistance, which should ultimately inproved cancer the rapies.

In recent research, Dr Yamaoka and a team at Showa University investigated the particular mechanisms that cause resistance to the third-generation EGFR TKI drugs rociletinib and osimertinib. Previously, the team had identified a specific genetic mutation in lung cancer cells that causes resistance to afatinib. In the subsequent study, they used cancer cells known to carry the afatinibresistance mutation to find out why resistance to rociletinib and osimertinib develops.

In this study, Dr Yamaoka used cells that were originally obtained from a lung cancer patient in the 1980s. To generate resistance to afatinib, the

cells were grown for several months in a medium that contained increasing concentrations of the drug. These cells were then subject to a repeat of the same process, but this time with either rociletinib or osimertinib in the growth medium, in order to trigger resistance to these medications. The team subsequently used various techniques, including genomic sequencing, to ascertain the genetic changes that had occurred in the two groups of drug-

\section{RESISTANCE REVEALED}

When they began this research, the team expected that resistance to rociletinib and

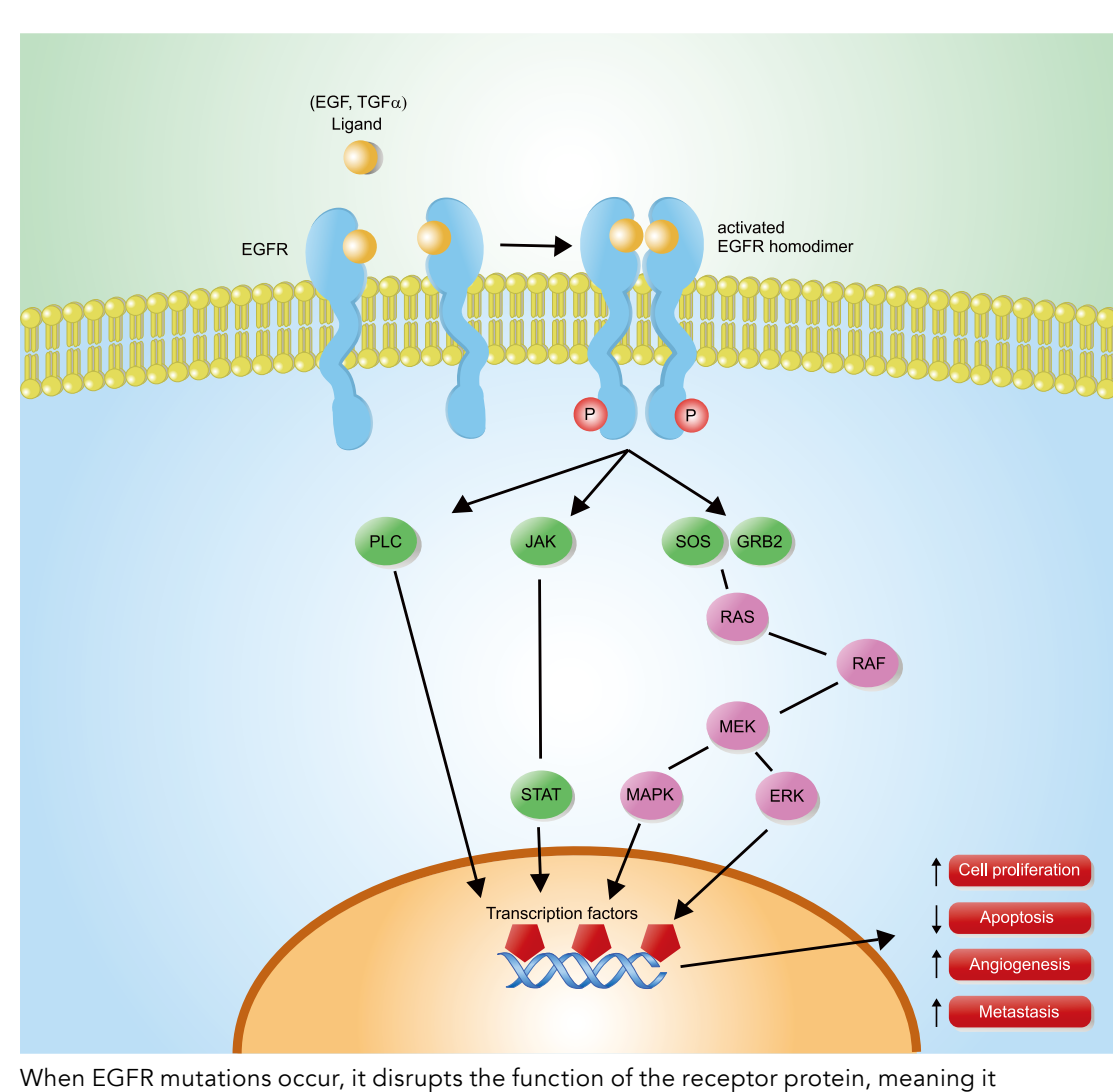

Their results revealed that resistance to rociletinib and osimertinib is indeed a result of unique genetic mutations.

osimertinib would be triggered by novel termed EGFR-T790M, that is linked genetic changes. This idea was based to non-small cell lung cancer. When on previous work, in which the team this mutation anises in cancerous cells, discovered three distinct mechanisms that lead to afatilib resistance in lung As Dr Yamaoka and his colleagues

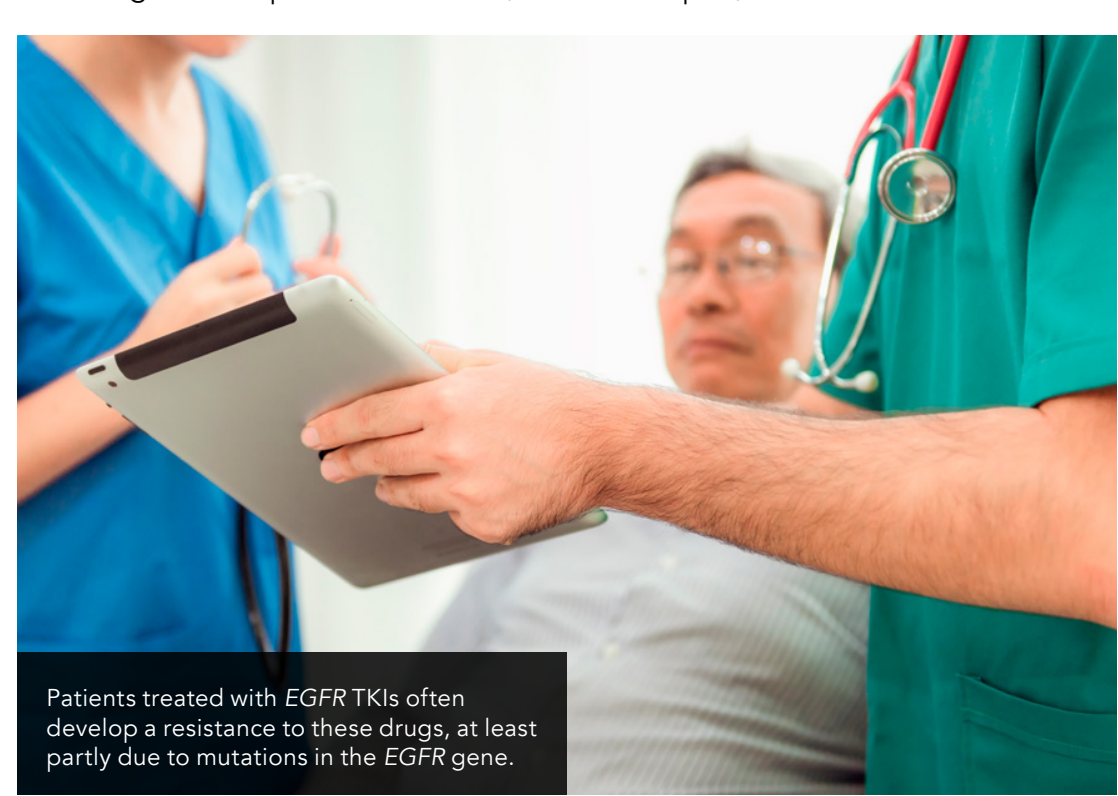




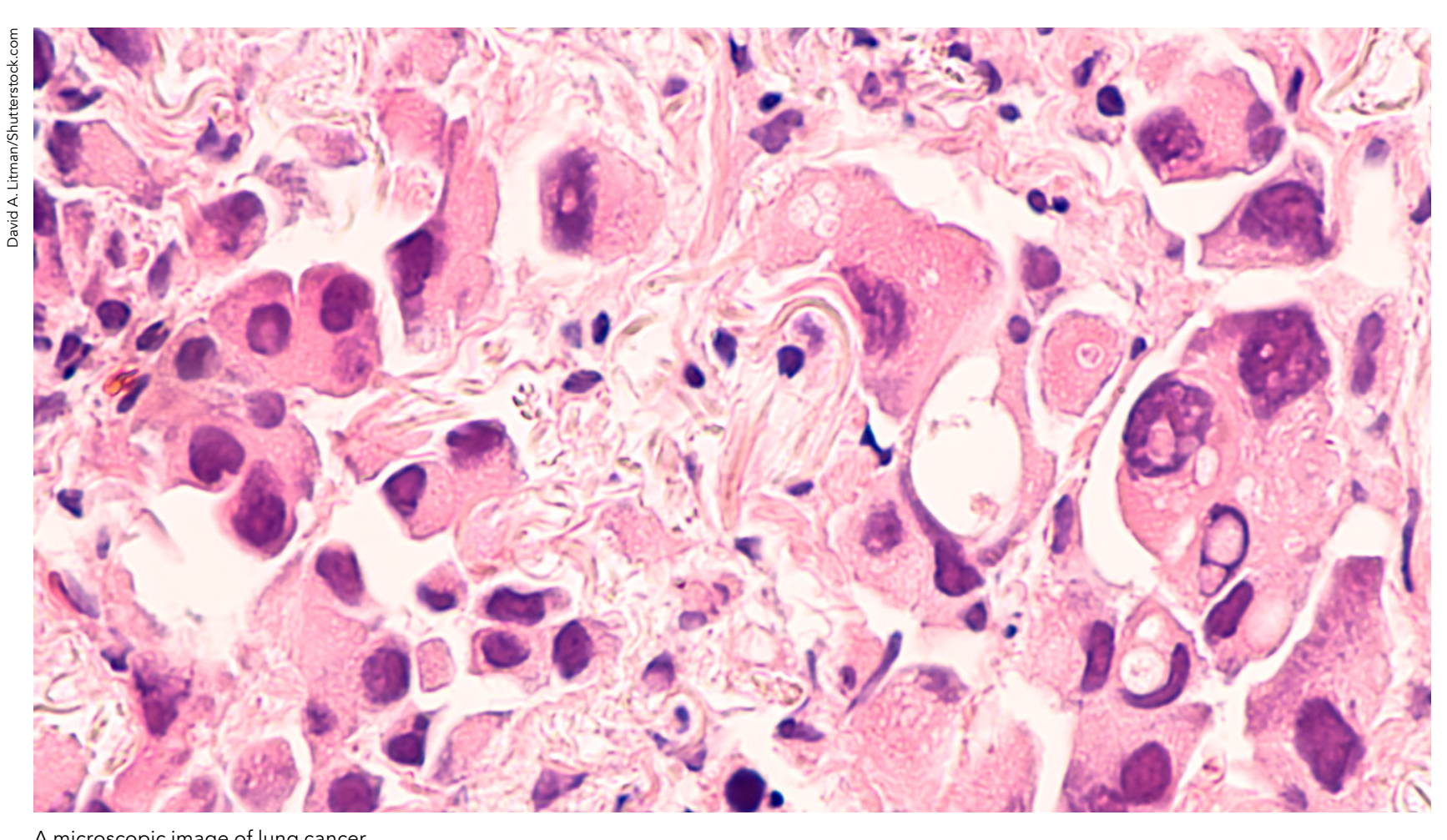

A microscopic image of lung cancer.

resistance to rociletinib and osimertinib is indeed a result of unique genetic

increase in the number of copies of a gene - was observed in both EGFR and $K R A S$, another gene involved in cell signalling, in the rociletinib-resistant
cells. Amplification and subsequent cells. Amplification and subsequent

after osimertinib was withdrawn, this protein complex broke down. This suggests that when patients develop resistance to osimertinib, the treatment could be withdrawn for two months; on being reinstated, the cancer should respond to the drug significant medical problem, particularly

(where genes

are activated

inappropriately,

as when EGFR

mutations cause

Importantly, in one group of osimertinibresistant cells, resistance was revealed

protein to be always 'on') has been could be responsible for a tumour

becoming malignant.

Dr Yamaoka also spotted KRAS amplification in the osimertinibresistant cells. The degree of resistance to osimertinib appeared to grow as the concentration of the drug was increased. Importantly, in one group of osimertinib-resistant cells, resistance was revealed to be reversible; when the drug was removed, KRAS activity was dulled, and the cells eventually regained sensitivity to osimertinib. In these found to be caused by a pas found to be caused by a particular

\section{to be reversible.}

\section{generation EGFR}

TKIs, rociletinib is acquired, $\mathrm{Dr}$ Yamaoka and his colleagues have along potential new treatment routes.

that this would be a better treatment option than continuous administration of EGFR TKIs.

Interpreting the results of this study has enabled Dr Yamaoka to suggest that an effective treatment for nonsmall cell lung cancer could be a combination of third-generation EGFR with another group of drugs called particular cell-signalling pathway tha particular cell-signalling pathway th results already suggest that osimertin following afatinib treatment greatly slows the progression of lung cancer. small cell lung cancer patients. However, such samples are difficult to take from the lungs without causing excessive damage. Another possibility would be circulating tumour DNA. These are DNA fragments that, having been shed from the primary and/or metastatic tumour, travel around the body in the blood - they are therefore much easier to sample than tissues deep within a core organ such as the lungs. This is the direction that Dr Yamaoka and his collegues will take, as they pursue new from non-small cell lung cancer. TKIs, like rociletinib and osimertinib,

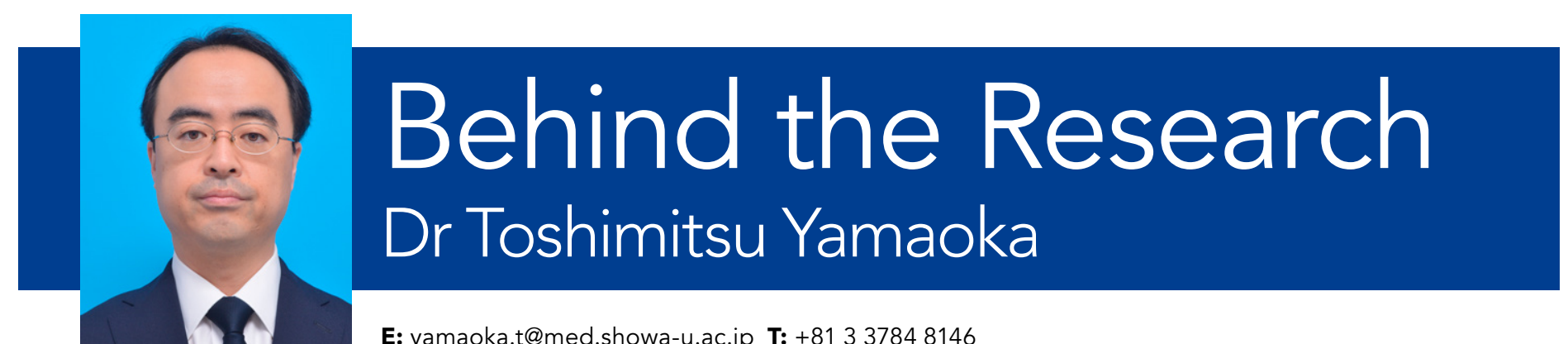

\section{FROM RESEARCH}

How to treat patients who have become resistant to EGFR TKI drugs represents for patients in the Asia-Pacific region including Japan. By successfully

describing how resistance to two third-

\section{Detail} to explore EGFR-TKI resistance using

\section{Research Objectives}

The clinical trial "The continuous evaluation of EGFR mutation in EGFR-mutation positive lung cancer patients during EGFR TKI treatment identifies novel resistance mechanisms in EGFR-mutation positive lung cancer treatments.

Toshimitsu Yamaoka

-5-8 Hatanodai

Shinagawa-ku

Tokyo

apan

Bio

DrYamaoka's medical career began at the Division of Allergology \& Respiratory Medicine at Showa University Hospital (Tokyo, Japan), providing medical services for patients with lung cancer, asthma, COPD, pneumonia and chronic bronchial infection. His research career started at the Institute of Molecular Oncology (now called the Advanced Cancer Translational Research Institute) from 1998. Shortly after, Yamaoka's mentor, Dr Ohmon, discovered an EGRR, activating mutation in tissue, malivaling them to discover the resistance mechanisms to EGFR TKIs.

\section{Funding}

\section{References}

Nakatani, K. et al. (2019). KRAS and EGFR amplifications mediate resistance to rociletinib and osimertinib in acquired

T790M in EGFR. Mol Cancer Ther, 18(1), 112-126.

\section{Personal Response}

How do you envision future treatment of EGFR TKI

II The therapies for patients with EGFR-activating with EGFR TKI to pursue longer survival and develop another concept to target EGFR-activating mutation. For the after second-line therapy, it is necessary clarify the various resistance pechanisms and to obtain therapeutic tools which can overcome the
corresponding resistance mechanisms, continuously.

of the Division of Allergology and Respiratory Medicine

Translational Research Institute at Showa University

\section{SHOWA University} mutations can improve as first-line therapy and after
second-line therapy. For the first-line therapy, we should find a more effective combination therapy

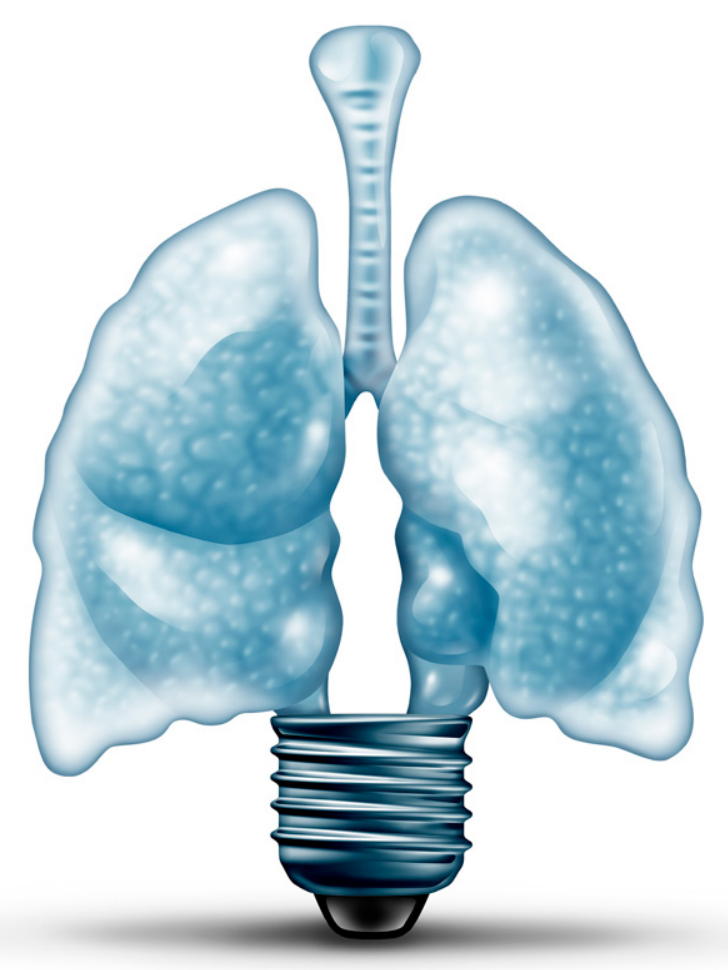

